Pacific Journal of Mathematic 


\title{
A BRANCHING LAW FOR THE SYMPLECTIC GROUPS
}

\author{
WiLlaRd MiLleR, JR.
}

\begin{abstract}
A "branching law" is derived for the irreducible tensor representations of the symplectic groups, and a relation is given between this law and the representation theory of the general linear groups.
\end{abstract}

Branching laws for the irreducible tensor representations of the general linear and orthogonal groups are well-known. Furthermore, these laws have a simple form [1]. In the case of the symplectic groups, however, the branching law becomes more complicated and is expressed in terms of a determinant. We derive this result here by brute force applied to the Weyl character formulas, though it could also have been obtained from a more sophisticated treatment of representation theory contained in some unpublished work of Kostant.

The Branching law. Let $V^{n}$ be an $n$-dimensional vector space over the complex field. The symplectic group in $n$ dimensions, $S_{p}(n / 2)$, is the set of all linear transformations $a \in \mathscr{E}\left(V^{n}\right)$, under which a nondegenerate skew-symmetric bilinear form on $V^{n} \times V^{n}$ is invariant, [3]. If $\langle\cdot, \cdot\rangle$ is the bilinear form on $V^{n} \times V^{n}$ and $a \in \mathscr{E}\left(V^{n}\right)$, then

$$
a \in S_{p}(n / 2) \text { if and only if }\langle a x, a y\rangle=\langle x, y\rangle \text { for all } x, y \in V^{n} \text {. }
$$

It is well-known that $S_{p}(n / 2)$ can be defined only for even dimensional spaces, $(n=2 \mu, \mu$ an integer). It is always possible to choose a basis $e_{i}, e_{i}^{\prime}, i=1, \cdots, \mu$ in $V^{n}$ such that

$$
\begin{aligned}
& \left\langle e_{i}, e_{j}\right\rangle=\left\langle e_{i}^{\prime}, e_{\jmath}^{\prime}\right\rangle=01 \leqq i, j \leqq \mu \\
& \left\langle e_{i}, e_{j}^{\prime}\right\rangle=\delta_{i j} .
\end{aligned}
$$

We assume that the matrix realization of $S_{p}(\mu)$ is given with respect to such a basis [3]. The unitary symplectic group, $U S_{p}(\mu)$, is defined by

$$
U S_{p}(\mu)=S_{p}(\mu) \cap U(2 \mu)
$$

where $U(2 \mu)$ is the group of unitary matrices in $2 \mu$ dimensions. The irreducible continuous representations of $U S_{p}(\mu)$ can be denoted by ${ }^{\mu} \omega_{f_{1}, \cdots, f_{\mu}}$, where $f_{1}, f_{2}, \cdots, f_{\mu}$ are integers such that $f_{1} \geqq f_{2} \geqq \cdots \geqq$ $f_{\mu-1} \geqq f_{\mu} \geqq 0$.

Received May 8, 1964. This work represents results obtained in part at the Courant Institute of Mathematical Sciences, New York University, under National Science Foundation grant GP-1669. 
Let $a \in U S_{p}(\mu)$. The eigenvalues of $a$ are

$$
\varepsilon_{1}, \varepsilon_{2}, \cdots, \varepsilon_{\mu}, \varepsilon_{1}^{-1}, \varepsilon_{2}^{-1}, \cdots, \varepsilon_{\mu}^{-1}
$$

where $\left|\varepsilon_{1}\right|=1,1 \leqq i \leqq \mu$, (see [3]). The character of the representation ${ }^{\mu} \omega_{f_{1}, \ldots, f_{\mu}}$ is given by

$$
{ }^{\mu} \chi_{f_{1}, \ldots, f_{\mu}}(a)=\frac{\left|\varepsilon^{l_{1}}-\varepsilon^{-l_{1}}, \varepsilon^{l_{2}}-\varepsilon^{-l_{2}}, \cdots, \varepsilon^{l \mu}-\varepsilon^{-l \mu}\right|}{\left|\varepsilon^{\mu}-\varepsilon^{-\mu}, \varepsilon^{\mu-1}-\varepsilon^{-\mu+1}, \cdots, \varepsilon-\varepsilon^{-1}\right|}
$$

$l_{i}=f_{i}+\mu-i+1,1 \leqq i \leqq \mu$.

The vertical lines in the numerator and denominator of (4) denote two determinants whose $j$ th rows are obtained by replacing the symbol $\varepsilon$ by $\varepsilon_{j}$.

Let ${ }^{\mu} \omega_{f_{1}, \ldots, f_{\mu}}$ and ${ }^{\mu-1} \omega_{g_{1}, \ldots, g_{\mu-1}}$ be irreducible representations of the groups $U S_{p}(\mu), U S_{p}(\mu-1)$, respectively. Denote by $R_{g_{1}, \ldots, g_{\mu-1}}^{f_{1}, \cdots, f_{\mu}}$ the the multiplicity of ${ }^{\mu-1} \omega_{g_{1}, \ldots, g_{\mu-1}}$ in the restricted representation ${ }^{\mu} \omega_{f_{1}, \ldots, f_{\mu}} /$ $U S_{p}(\mu-1)$ which is obtained by restricting ${ }^{\mu} \omega$ to the subgroup of $U S_{p}(\mu)$ consisting of all matrices that leave the basis vectors $e_{\mu}, e_{\mu}^{\prime}$ fixed.

THEOREM 1. $\quad R_{g_{1}, \ldots, g_{\mu-1}}^{f_{1}, \ldots, f_{\mu}}=0$ unless $f_{i} \geqq g_{i} \geqq f_{i+2}, 1 \leqq i \leqq \mu-2$. If these conditions are fulfilled, then

(5) $R_{g_{1}, \cdots, g_{\mu-1}}^{f_{1}, \cdots, \mu}$

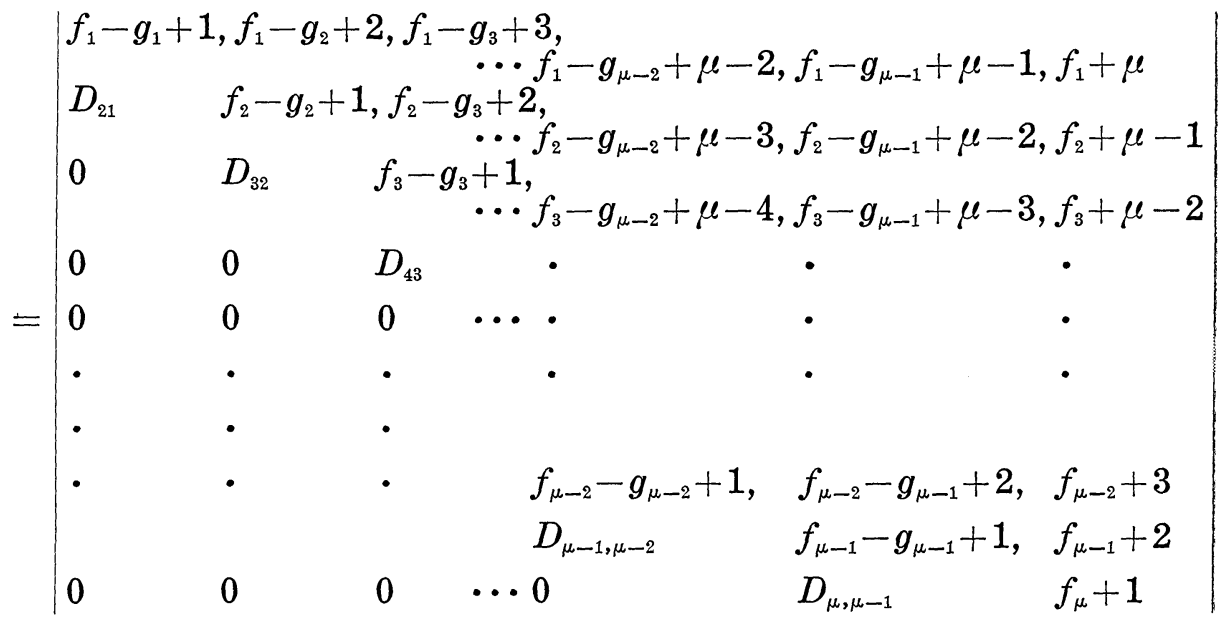

where $D_{i+1, i}=\max \left[f_{i+1}-g_{i}, 0\right], 1 \leqq i \leqq \mu-1$.

Proof. The dependence of the character of the representation ${ }^{\mu} \omega_{f_{1}, \cdots, f_{\mu}}$ on the eigenvalues $\varepsilon_{1}, \cdots, \varepsilon_{\mu}$ of $a \in U S_{p}(\mu)$ can be explicitly exhibited by setting

$$
{ }^{\mu} \chi_{f_{1}, \ldots, f_{\mu}}(a)={ }^{\mu} \chi_{f_{1}, \ldots, f_{\mu}}\left(\varepsilon_{1}, \cdots, \varepsilon_{\mu}\right) .
$$


The restriction of ${ }^{\mu} \omega_{f_{1}, \ldots, f_{\mu}}$ to $U S_{p}(\mu-1)$ can be accomplished by requiring that $\varepsilon_{\mu}=1$. It follows that

$$
\begin{aligned}
& { }^{\mu} \chi_{f_{1}, \ldots, f_{\mu}}\left(\varepsilon_{1}, \cdots, \varepsilon_{\mu-1}, 1\right) \\
& =\sum R_{g_{1}, \cdots, g_{\mu-1}}^{f_{1}, \cdots, \chi_{g_{1}}, \cdots, g_{\mu-1}}\left(\varepsilon_{1}, \cdots, \varepsilon_{\mu-1}\right) \\
& \quad g_{1}, \cdots, g_{\mu-1} .
\end{aligned}
$$

We will calculate the constants $R_{g_{1}, \ldots, g_{\mu-1}}^{f_{1}, \ldots, f \mu}$ by carrying out the decomposition in equation (6). If we take the limit as $\varepsilon_{\mu} \rightarrow 1$ in the character formula (4), we get

$$
\begin{aligned}
& { }^{\mu} \chi_{f_{1}, \ldots, f_{\mu}}\left(\varepsilon_{1}, \cdots, \varepsilon_{\mu-1}, 1\right)
\end{aligned}
$$

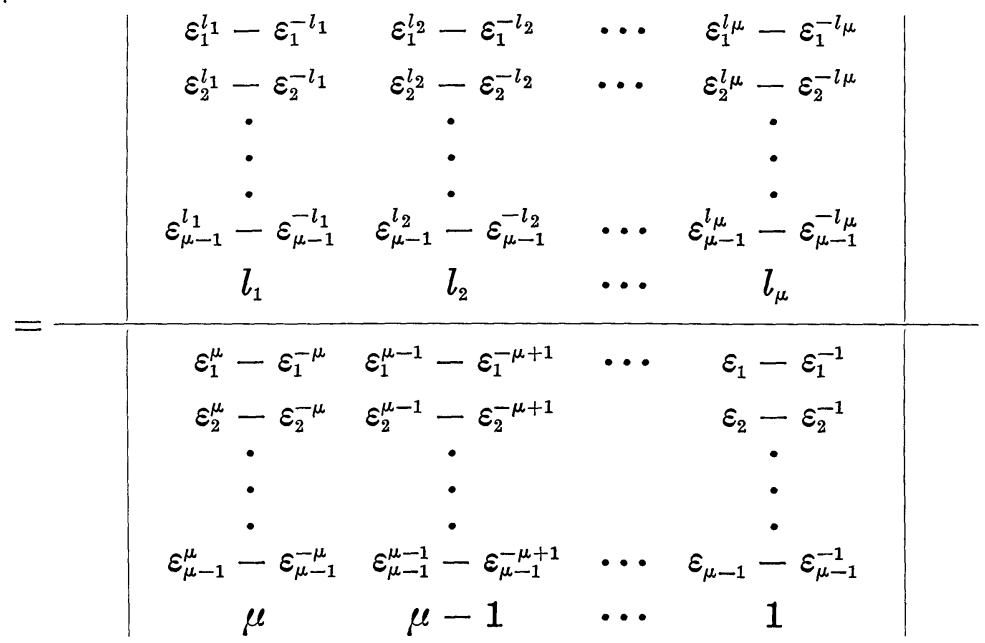

Set $s_{i}(j)=\left(\varepsilon_{i}\right)^{j}-\left(\varepsilon_{i}\right)^{-j}, 1 \leqq i \leqq \mu-1$

$$
d_{i}=\varepsilon_{i}+\varepsilon_{i}^{-1}-2,1 \leqq i \leqq \mu-1 .
$$

It is easy to verify the formula

$$
s_{i}(n-1) d_{i}=s_{i}(n)-2 s_{i}(n-1)+s_{i}(n-2) .
$$

Also, the relation

$$
s_{i}(n)=d_{i}\left[s_{i}(n-1)+2 s_{i}(n-2)+\cdots+k s_{i}(n-k)+\cdots+(n-1) s_{i}(1)\right]+n s_{i}(1)
$$

can be established by induction on (8).

Consider the determinant in the denominator of equation (7). Using obvious abbreviations, we have

$$
\left|\begin{array}{c}
s(\mu), s(\mu-1), \cdots s(2), s(1) \\
\mu, \mu-1, \cdots 2,1
\end{array}\right|=
$$




$$
\begin{aligned}
& =\mid s(\mu)-s(\mu-1), s(\mu-1)-s(\mu-2), \cdots, s(2)-s(1), s(1) \\
& 1 \quad \begin{array}{ccc}
1 & \cdots, & 1
\end{array} \mid \\
& =\mid s(\mu)-2 s(\mu-1)+s(\mu-2), s(\mu-1)-2 s(\mu-2)+s(\mu-3), \\
& 0 \quad, \quad \cdots, s(2)-2 s(1), s(1) \\
& =|s(\mu)-2 s(\mu-1)+s(\mu-2), s(\mu-1)-2 s(\mu-2)+s(\mu-3), \cdots, s(2)-2 s(1)|^{\prime} \\
& =\prod_{i=1}^{\mu-1} d_{i}|s(\mu-1), s(\mu-2), \cdots, s(2), s(1)|^{\prime} .
\end{aligned}
$$

Equation (8) was used in the last step of (10). The quantity $|\cdot|^{\prime}$ stands for a determinant of order $\mu-1$.

Now, consider the numerator of (7).

We have

$$
\begin{aligned}
& \mid s\left(l_{1}\right),\left(s\left(l_{2}\right), \cdots, s\left(l_{\mu}\right) \mid=\text { (using }(9)\right) \\
& l_{1}, l_{2}, \cdots, l_{\mu} \\
& =\mid d\left[s\left(l_{1}-1\right)+\cdots+\left(l_{1}-1\right) s(1)\right]+l_{1} s(1), \cdots, d\left[s\left(l_{\mu}-1\right)+\cdots+\left(l_{\mu}-1\right) s(1)\right] \\
& l_{1}, \cdots, \quad l_{\mu} \\
& =l_{\mu} \mid d\left\{\left[s\left(l_{1}-1\right)+\cdots+\left(l_{1}-1\right) s(1)\right]-\frac{l_{1}}{l_{\mu}}\left[s\left(l_{\mu}-1\right)+\cdots+\left(l_{\mu}-1\right) s(1)\right]\right\}, \cdots \\
& \cdots, d\left\{\left[s\left(l_{\mu-1}-1\right)+\cdots+\left(l_{\mu-1}-1\right) s(1)\right]\right. \\
& \left.-\frac{l_{\mu-1}}{l_{\mu}}\left[s\left(l_{\mu}-1\right)+\cdots+\left(l_{\mu}-1\right) s(1)\right]\right\}\left.\right|^{\prime} \text {. }
\end{aligned}
$$

Set $q_{j}(i)=s_{j}\left(l_{i}-1\right)+2 s_{j}\left(l_{i}-2\right)+\cdots+\left(l_{i}-1\right) s_{j}(1), 1 \leqq i \leqq \mu, 1 \leqq$ $j \leqq \mu-1$. Then, we find that the numerator of (7) is equal to

$$
\begin{aligned}
& l_{\mu} \prod_{i=1}^{\mu-1} d_{i}\left|q(1)-\frac{l_{1}}{l_{\mu}} q(\mu), q(2)-\frac{l_{2}}{l_{\mu}} q(\mu), \cdots, q(\mu-1)-\frac{l_{\mu-1}}{l_{\mu}} q(\mu)\right|^{\prime} \\
& =\prod_{i=1}^{\mu-1} d_{i}\left\{l_{\mu}|q(1), q(2), \cdots, q(\mu-1)|^{\prime}\right. \\
& -l_{1}|q(\mu), q(2), q(3), \cdots, q(\mu-1)|^{\prime} \\
& -l_{2}|q(1), q(\mu), q(3), \cdots, q(\mu-1)|^{\prime} \\
& \left.-\cdots-l_{\mu-1}|q(1), q(2), \cdots, q(\mu-2), q(\mu)|^{\prime}\right\} .
\end{aligned}
$$

Dividing the last expression in (12) by the last expression in (10), we cancel the factor $\prod_{i=1}^{\mu-1} d_{i}$. Thus, to calculate $R_{g_{1}, \ldots, g_{\mu-1}}^{f_{1}, \ldots, f_{\mu}}$ it only remains to expand the determinants in (12) as linear combinations of determinants of the form

$$
\left|s\left(h_{1}\right), s\left(h_{2}\right), \cdots, s\left(h_{\mu-1}\right)\right|^{\prime}, h_{1}>\cdots>h_{\mu-1}>0 \text {. }
$$

Set $p_{i}=g_{i}+\mu-i, \quad 1 \leqq i \leqq \mu-1$. Then $R_{g_{1}, \cdots, g_{\mu-1}}^{f_{1}, \cdots, f}$ will be the 
coefficient of the determinant

$$
\left|s\left(p_{1}\right), s\left(p_{2}\right), \cdots, s\left(p_{\mu-1}\right)\right|^{\prime}
$$

in the expansion of (12). It is straightforward to show that

$$
R_{g_{1}, \cdots, g_{\mu-1}}^{f_{1}, \cdots, f \mu}=\sum_{\sigma} \operatorname{sgn} \sigma\left\langle l_{\sigma(1)}-p_{1}\right\rangle\left\langle l_{\sigma(2)}-p_{2}\right\rangle \cdots\left\langle l_{\sigma(\mu-1)}-p_{\mu-1}\right\rangle l_{\sigma(\mu)}
$$

where the sum is taken over all permutations $\sigma$ of the integers $1,2, \cdots, \mu$. The quantity

$$
\left\langle l_{i}-p_{j}\right\rangle=\left\{\begin{array}{cc}
l_{i}-p_{j} & \text { if } l_{i}-p_{j} \geqq 0 \\
0 & \text { if } l_{i}-p_{j}<0
\end{array} .\right.
$$

Thus,

$$
R_{g_{1}, \cdots, g_{\mu-1}}^{f_{1}, \cdots, f_{\mu}}=\left|\begin{array}{cccc}
\left\langle l_{1}-p_{1}\right\rangle, & \left\langle l_{1}-p_{2}\right\rangle & \cdots\left\langle l_{1}-p_{\mu-1}\right\rangle, & l_{1} \\
\left\langle l_{2}-p_{1}\right\rangle, & \left\langle l_{2}-p_{2}\right\rangle & \cdots\left\langle l_{2}-p_{\mu-1}\right\rangle, & l_{2} \\
\cdot & \dot{\cdot} & \dot{\bullet} & \dot{\cdot} \\
\left\langle l_{\mu}-p_{1}\right\rangle, & \left\langle l_{\mu}-p_{2}\right\rangle & \cdots\left\langle l_{\mu}-p_{\mu-1}\right\rangle, & l_{\mu}
\end{array}\right|
$$

An analysis of expression (16) yields the theorem.

CoROLLARY. $\quad R_{g_{1}, \ldots, g_{\mu-2}, 0}^{f_{1}, \ldots, f_{\mu-1}, 0}=R_{g_{1}, \ldots, g_{\mu-2}}^{f_{1}, \ldots, f_{\mu-1}}$

Proof. Direct verification from expression (5).

It is well-known that the continuous irreducible representations of the $n \times n$ unitary group $U(n)$ can be denoted by ${ }^{n} \nu_{f_{1}}, \ldots, f_{n}$ where the integers $f_{1}, f_{2}, \cdots, f_{n}$ can take on all values consistent with $f_{1} \geqq f_{2} \geqq$ $\cdots f_{n}$, [3]. We make the assumption that $f_{n} \geqq 0$.

$U(n)$ contains a subgroup $G(n-2)=U(n-2)+E_{2}$ where $E_{2}$ is the $2 \times 2$ unit matrix, which is obviously isomorphic to $U(n-2)$. (see [1], page 16 for the notation). We identify $G(n-2)$ and $U(n-$ 2) by this isomorphism. Thus the irreducible continuous representations of $G(n-2)$ will be denoted by ${ }^{n-2} \nu_{g_{1}, \ldots, g_{n-2}}$.

Denote by $M_{g_{1}, \cdots, g_{n-2}}^{f_{1}, \ldots, f_{n}}$ the multiplicity of ${ }^{n-2} \nu_{g_{1}, \ldots, g_{n-2}}$ in the restricted representation ${ }^{n} \nu_{f_{1}}, \ldots, f_{n} / G(n-2)$. The quantity $M_{g_{1}, \cdots, g_{n-2}}^{f_{1}, \ldots, f_{n}}$ can be computed from the Weyl character formula for the irreducible representations of $U(n)$ in the same way as we have done for the irreducible representations of $U S_{p}(\mu)$. We give only the results of this computation.

THEOREM 2. Let $M_{g_{1}, \cdots, g_{\mu-1}}^{f_{1}, \ldots, f_{\mu+1}}$ be the multiplicity of ${ }^{\mu-1} \nu_{g_{1}, \ldots, g_{\mu-1}}$ in ${ }^{\mu+1} \nu_{f_{1}, \ldots, f_{\mu+1}} / U(\mu-1)$ as defined above. 


$$
M_{g_{1}, \ldots, g_{\mu-1}}^{f_{1}, \ldots, f_{\mu, 0}}=R_{g_{1}, \ldots, g_{\mu-1}}^{f_{1}, \ldots, f_{\mu}}
$$

CoROLLARY. $\quad M_{g_{1}, \cdots, g_{\mu-2}}^{f_{1}, \ldots, f_{\mu}}=R_{g_{1}, \cdots, f_{-2}, 0}^{f_{1}, \ldots, f_{\mu}}$

\section{REFERENCES}

1. H. Boerner, Representations of Groups, North Holland, Amsterdam. 1963.

2. W. Miller, On a class of vector-valued functions covariant under the classical groups with applications to physics, National Science Foundation Technical Report. University of California, Berkeley. 1963.

3. H. Weyl, The Classical Groups. Princeton University Press, Princeton. 1946.

Courant Institute of Mathematical Sciences

NEW YORK UNIVERSITY 


\section{PACIFIC JOURNAL OF MATHEMATICS}

\section{EDITORS}

\section{H. SAMELSON}

Stanford University

Stanford, California

\section{R. M. BLUMenthaL \\ University of Washington \\ Seattle, Washington 98105}

*J. DugundjI

University of Southern California Los Angeles, California 90007

\section{RICHARD ARENS}

University of California

Los Angeles, California 90024

\section{ASSOCIATE EDITORS}
E. F. BECKENBACH
B. H. NeUManN
F. WOLF
K. YosIDA

\section{SUPPORTING INSTITUTIONS}

\author{
UNIVERSITY OF BRITISH COLUMBIA \\ CALIFORNIA INSTITUTE OF TECHNOLOGY \\ UNIVERSITY OF CALIFORNIA \\ MONTANA STATE UNIVERSITY \\ UNIVERSITY OF NEVADA \\ NEW MEXICO STATE UNIVERSITY \\ OREGON STATE UNIVERSITY \\ UNIVERSITY OF OREGON \\ OSAKA UNIVERSITY \\ UNIVERSITY OF SOUTHERN CALIFORNIA
}

\author{
STANFORD UNIVERSITY \\ UNIVERSITY OF TOKYO \\ UNIVERSITY OF UTAH \\ WASHINGTON STATE UNIVERSITY \\ UNIVERSITY OF WASHINGTON \\ AMERICAN MATHEMATICAL SOCIETY \\ CHEVRON RESEARCH CORPORATION \\ TRW SYSTEMS \\ NAVAL ORDNANCE TEST STATION
}

Mathematical papers intended for publication in the Pacific Journal of Mathematics should be typewritten (double spaced). The first paragraph or two must be capable of being used separately as a synopsis of the entire paper. It should not contain references to the bibliography. Manuscripts may be sent to any one of the four editors. All other communications to the editors should be addressed to the managing editor, Richard Arens at the University of California, Los Angeles, California 90024.

50 reprints per author of each article are furnished free of charge; additional copies may be obtained at cost in multiples of 50 .

The Pacific Journal of Mathematics is published monthly. Effective with Volume 16 the price per volume (3 numbers) is $\$ 8.00$; single issues, $\$ 3.00$. Special price for current issues to individual faculty members of supporting institutions and to individual members of the American Mathematical Society: $\$ 4.00$ per volume; single issues $\$ 1.50$. Back numbers are available.

Subscriptions, orders for back numbers, and changes of address should be sent to Pacific Journal of Mathematics, 103 Highland Boulevard, Berkeley 8, California.

Printed at Kokusai Bunken Insatsusha (International Academic Printing Co., Ltd.), No. 6, 2-chome, Fujimi-cho, Chiyoda-ku, Tokyo, Japan.

PUBLISHED BY PACIFIC JOURNAL OF MATHEMATICS, A NON-PROFIT CORPORATION

The Supporting Institutions listed above contribute to the cost of publication of this Journal, but they are not owners or publishers and have no responsibility for its content or policies.

* Paul A. White, Acting Editor until J. Dugundji returns. 


\section{Pacific Journal of Mathematics}

\section{Vol. 16, No. 2 December, 1966}

Loren N. Argabright, Invariant means on topological semigroups ........ 193

William Arveson, A theorem on the action of abelian unitary groups ...... 205

John Spurgeon Bradley, Adjoint quasi-differential operators of Euler

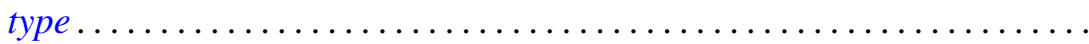

Don Deckard and Lincoln Kearney Durst, Unique factorization in power series rings and semigroups ........................... 239

Allen Devinatz, The deficiency index of ordinary self-adjoint differential operators..................................... 243

Robert E. Edwards, Operators commuting with translations ............ 259

Avner Friedman, Differentiability of solutions of ordinary differential equations in Hilbert space .............................. 267

Boris Garfinkel and Gregory Thomas McAllister, Jr., Singularities in a variational problem with an inequality ......................

Seymour Ginsburg and Edwin Spanier, Semigroups, Presburger formulas,

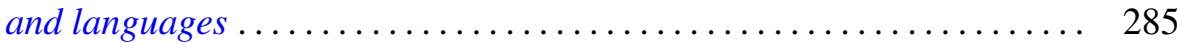

Burrell Washington Helton, Integral equations and product integrals . . . . . . 297

Edgar J. Howard, First and second category Abelian groups with the n-adic topology.........................................

Arthur H. Kruse and Paul William Liebnitz, Jr., An application of a family homotopy extension theorem to ANR spaces.

Albert Marden, I. Richards and Burton Rodin, On the regions bounded by homotopic curves

Willard Miller, Jr., A branching law for the symplectic groups ...

Marc Aristide Rieffel, A characterization of the group algebras of the finite

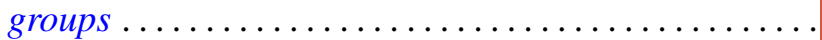

P. P. Saworotnow, On two-sided $H^{*}$-algebras

John Griggs Thompson, Factorizations of p-solvable groups ...

Shih-hsiung Tung, Harnack's inequalities on the classical Cartan

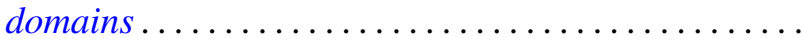

